\title{
EVALUACIÓN DE COMUNIDADES DE MACROINVERTEBRADOS ASOCIADOS A TRES ESPECIES DE MACRÓFITAS ACUÁTICAS EN LA LAGUNA DE MORONACOCHA, IQUITOS
}

\author{
Zoila Perea Saavedra,* Ivonne Bocanegra Chung y Miriam Alvan Aguilar \\ Facultad de Ciencias e Ingeniería, Universidad Científica del Perú, Av. Abelardo Quiñones Km 2.5, Iquitos, Perú. \\ e-mail: zoilitaperea@gmail.com
}

\begin{abstract}
RESUMEN
El objetivo fue evaluar la riqueza y abundancia de macroinvertebrados acuáticos asociados a tres especies de macrófitas acuáticas del Lago Moronacocha. Los muestreos se realizaron entre los meses de mayo y julio del 2009; se realizaron colectas mensuales de 27 muestras de cada macrófita, tomándose adicionalmente medidas de parámetros fisicoquímicos: temperatura, oxígeno y $\mathrm{pH}$. Las muestras de Pistia stratiotes (huama) y Echinocloa crassipes (putu putu), fueron colectadas con una red de bentos ( $20 \times 30 \mathrm{~cm}$ y $180 \mu \mathrm{m}$ de abertura de malla), mientras que los ejemplares de Echinocloa polystachya (gramalote) fueron colectados manualmente.

No se encontraron diferencias estadísticas significativas en cuanto la riqueza y abundancia entre las tres macrófitas evaluadas $(P>0.05)$. Se encontró una abundancia de 1538 individuos, siendo las familias más abundantes en la colecta: Chironomidae, Noteridae y Haplotaxidae, siendo además Hydrophilidae, Elmidae y Planorbidae abundantes solo para Pistia stratiotes. De las 31 familias reportadas en este estudio, 17 estuvieron presentes en las tres macrófitas evaluadas. Los valores promedio de $\mathrm{T}^{\circ}\left(26.4^{\circ} \mathrm{C}\right)$ y $\mathrm{pH}(6.7)$ registrados se encontraron dentro de los límites adecuados. Concluyendo que la composición de macroinvertebrados acuáticos en el Lago Moronacocha, no está influenciada por el tipo de macrófita que existe en ella.
\end{abstract}

Palabras clave: macrófitas, macroinvertebrados, abundancia y ocurrencia de Taxa

\section{EVALUATION OF MACROINVERTEBRATE COMMUNITIES ASSOCIATED WITH THREE SPECIES OF AQUATIC MACROPHYTES IN THE LAGOON OF MORONACOCHA, IQUITOS}

\begin{abstract}
The aim was to evaluate the richness and abundance of macroinvertebrates associated with three aquatic species of macrophytes of Lake Moronacocha. Sampling was conducted between May and July 2009, monthly collections of 27 samples of each macrophyte were made. Additionally, we took physicochemical measures such as: temperature, oxygen and $\mathrm{pH}$. Samples of Pistia stratiotes (huama) and Echinocloa crassipes (putu putu) were collected with a net of Benthos $(20 \times 30 \mathrm{~cm}$ and 180 um mesh net), while Echinocloa polystachya (gramalote) were collected manually.

There were no statistically significant differences among richness and abundance of the three macrophytes evaluated ( $P>0.05$ ). We found an abundance of 1538 individuals, being the most abundant families in the collection: Chironomidae, Noteridae y Haplotaxidae. Hydrophilidae, Elmidae y Planorbidae families were abundant only for Pistia stratiotes. Of the 31 families reported in this study, 17 were present in the three macrophytes evaluated. The average values of $\mathrm{T}^{\circ}\left(26.4^{\circ} \mathrm{C}\right)$ and $\mathrm{pH}(6.7)$ were found within the appropriate limits. Therefore, we conclude that composition of aquatic macroinvertebrates in the Lake Moronacocha, was not influenced by the type of macrophyte it contains.
\end{abstract}

Keywords: macrophytes, macroinvertebrates, abundance and occurrence of Taxa 


\section{INTRODUCCIÓN}

La laguna de Moronacocha, ubicada en la zona oeste de la ciudad de Iquitos, se caracteriza por presentar inundación periódica (Encarnación et al., 1985), influenciada directamente por la creciente y vaciante de las aguas del río Nanay. Este cuerpo de agua ha tenido y tiene múltiples usos para las poblaciones asentadas en su área de influencia, entre los que destacan el uso doméstico (bebida, cocina, aseo), el uso recreativo y el sustento diario a través de la pesca artesanal.

Sin embargo, a lo largo de las últimas décadas, la laguna Moronacocha ha venido atravesando perturbaciones de tipo antrópico, referidos en concreto a que su cubeta de agua se constituye en el depósitos de cuatro principales desagües de la ciudad de Iquitos y de los desechos arrojados por la población, ubicada en los alrededores del lago, esto propicia una creciente contaminación orgánica en sus aguas, lo que probablemente esté originando cambios físicos - químicos que afectarían de manera directa a las comunidades biológicas que en ellas viven (Araujo et al., 2003).

En la Amazonía peruana, los estudios que nos brinden conocimientos sobre la composición de macroinvertebrados asociados a macrófitas acuáticas, es insuficiente, teniendo en cuenta la importancia que tienen estos organismos en la evaluación de la calidad de aguas y su asociación con las macrófitas, en el entendimiento de la estructura y funcionamiento de los ecosistemas lacustres. El objetivo de la presente investigación es contribuir al conocimiento de las comunidades de macroinvertebrados asociados a tres especies de macrófitas acuáticas, como base preliminar para futuros estudios e inferir su uso como nuevos indicadores ecológicos.

\section{MATERIAL Y MÉTODOS}

\section{Área de estudio.}

Este estudio fue realizado en la laguna de Moronacocha, que se encuentra ubicado al lado oeste de la ciudad de Iquitos, entre las coordenadas geográficas $73^{\circ} 15^{\prime} 53.1^{\prime}$ ' LO y $3^{\circ} 44^{\prime} 52.4^{\prime}$ ' LE. Esta laguna posee una forma semilunar con una longitud aproximada de $8 \mathrm{Km}$, presentando una superficie aproximada de $200 \mathrm{Ha}$ (Carey et al., 1979).

\section{Colecta de muestras}

Los muestreos de las tres especies de macrófitas fueron realizados en un periodo de tres meses (mayo, junio y julio del 2009) entre las 8:00 y 12:00 horas. Se consideraron 9 puntos de colecta al azar, cuyas coordenadas geográficas son: 1) X:0692487; Y:9585866 2) X:0692484; Y:9585828 3) $\mathrm{X}: 0692609$; $\mathrm{Y:9585939}$ 4) X:0344827; Y:7316442 5) X:0344792; Y:7316450 6) X:0344797; Y:7316240 7) X:0344054; Y:7316319 8) X:0344058; Y:7316316 9) X:0344093; Y:7316224 por cada punto de colecta se realizaron 3 repeticiones, haciendo un total de 27 muestras por cada mes, teniendo un total de 81 muestras entre los tres meses de colectas. 


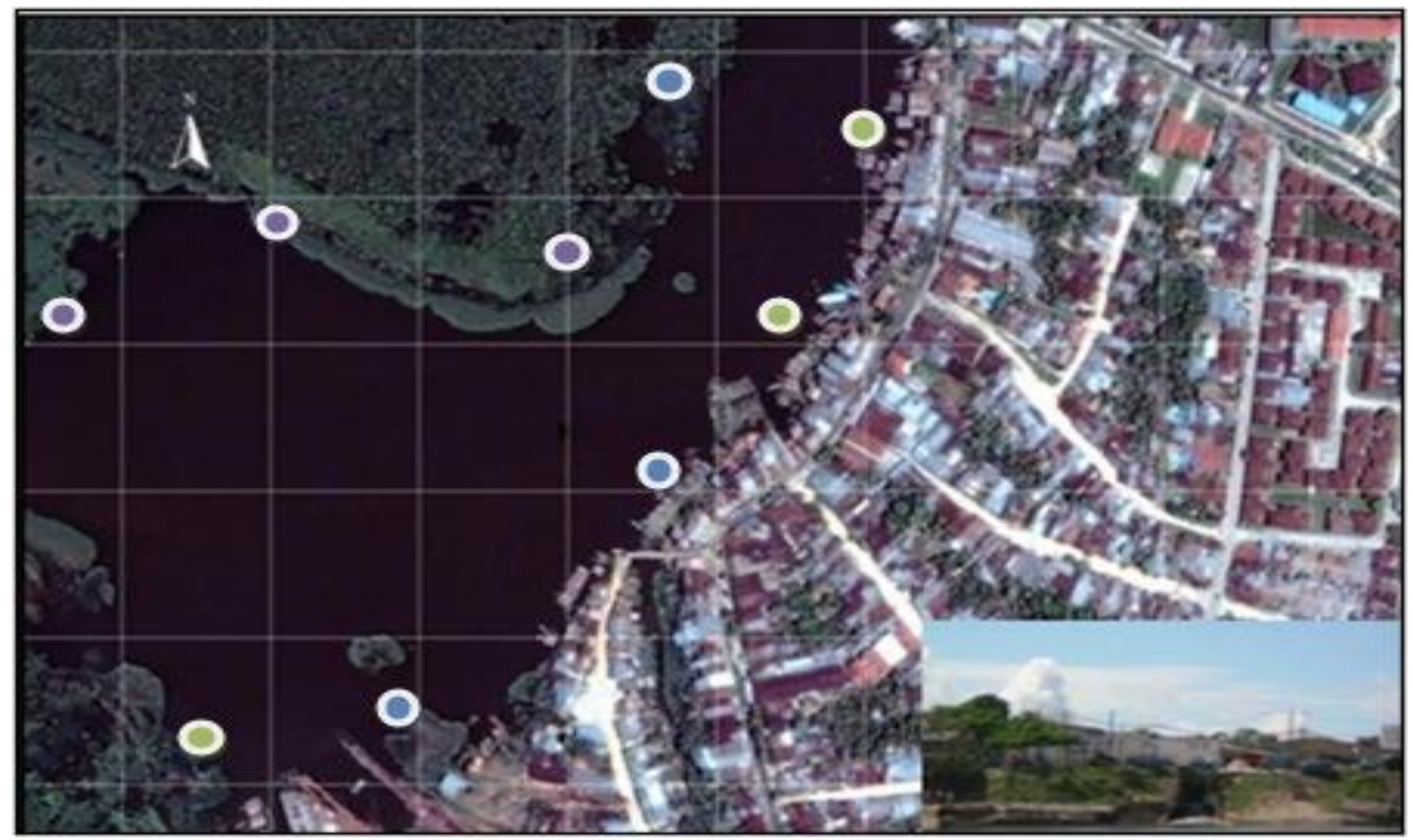

Figura 01. Lugares de muestreo en la laguna de Moronacocha, Iquitos- Perú. - Eichhornia crassipes (putu putu) - Pistia stratiotes (huama) Echinocloa polystachya (gramalote)

\section{Determinación de Riqueza y abundancia de macroinvertebrados acuáticos}

La riqueza y abundancia de macroinvertebrados acuáticos se identificaron en el Laboratorio de Taxonomía de Peces del Centro de Investigaciones Quistococha del Instituto de Investigaciones de la Amazonia Peruana (IIAP), La identificación de los macroinvertebrados fue realizada hasta los niveles taxonómicos de orden y familia, para eso se conto con la ayuda de las claves taxonómicas de MacCafferty \& Provonsha (1981), Merrit \& Cummins (1984), Roldan (1996) y Tachet (2000).

Por cada punto de colecta se tomaron registros de los parámetros físico - químicos: La temperatura y el $\mathrm{pH}$ fueron medidos utilizando el $\mathrm{pH}$-metro WTW pH330i, mientras que para el oxígeno disuelto se utilizó el oxímetro YSI Model 55.

\section{RESULTADOS}

\section{Riqueza de macroinvertebrados acuáticos.}

Se registraron un total de 31 familias de macroinvertebrados acuáticos, distribuidos en once órdenes (Coleoptera, Diptera, Ephemeroptera, Heteróptera, Odonata, Trichoptera, Lepidoptera, Conchostraca, Basommathopora, Glossiphoniiforme y Haplotaxida), cinco clases (Insecta, Branchiopoda, Gasterópoda, Hirudínea y Oligochaeta) y en tres phyla (Artrópoda, Molusca y Anélida).

De las cinco clases taxonómicas de macroinvertebrados acuáticos reportadas en este estudio, la clase Insecta fue la que presentó mayor riqueza de familias en las tres macrófitas evaluadas $(P$. stratiotes $=21, E$. crassipes $=18$ y $E$. polystachya=22), a diferencia de las clases Branchiopoda, Hirudinea y Oligochaeta que solo presentaron una familia para las tres macrófitas y la clase Gasterópoda quien estuvo presente solo en $P$. stratiotes con una única familia. La riqueza total de macroinvertebrados en las tres macrófitas evaluadas fueron las siguientes: $P$. stratiotes $=25, E$. crassipes $=21$ y $E$. polystachya=25 (Figura 02). 


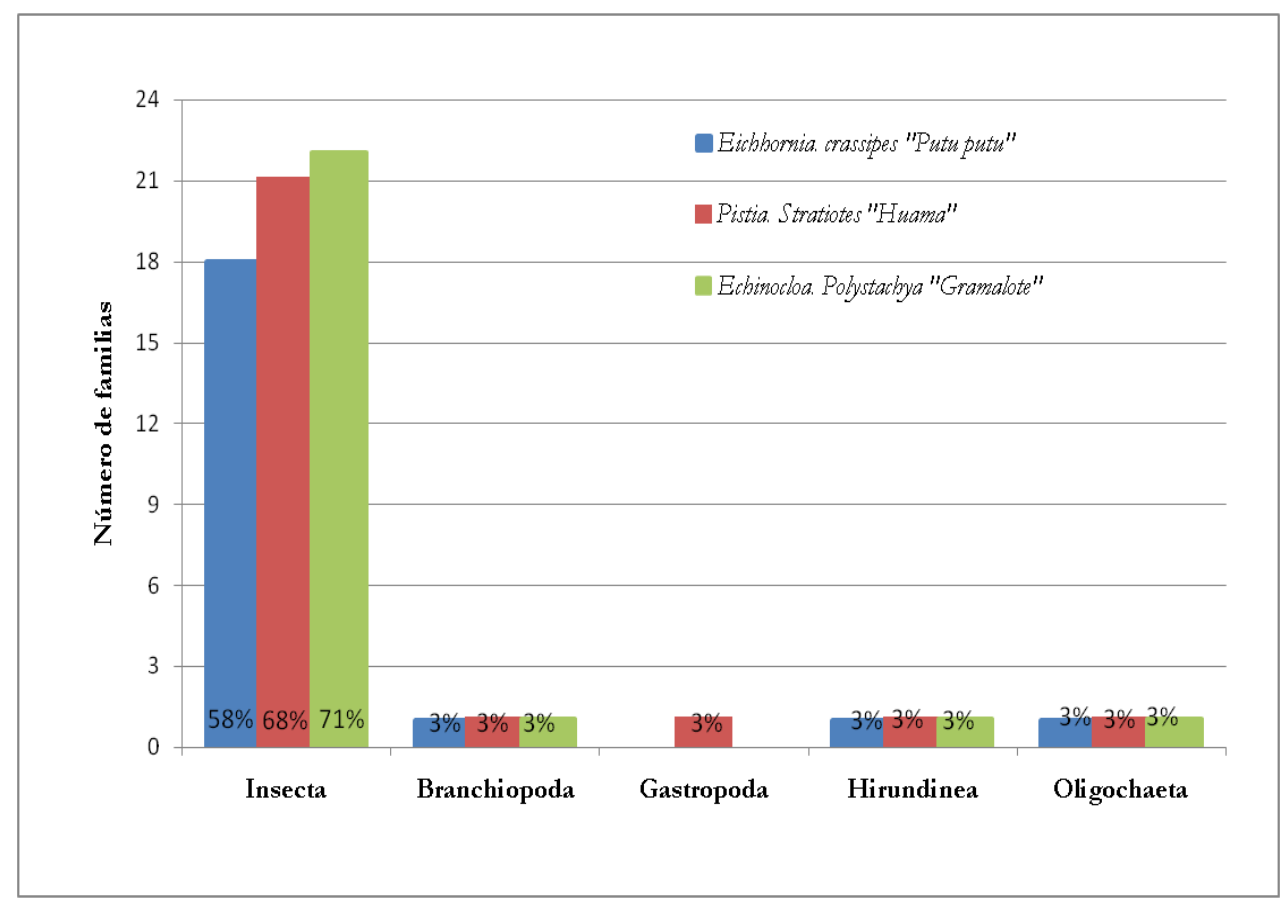

Figura 02. Riqueza total de familias de macroinvertebrados acuáticos por clase taxonómica y tipo de macrófita en la laguna de Moronacocha. (Iquitos- Perú).

El análisis de varianza indica que no hay diferencias estadísticas significativas en cuanto a la riqueza entre las tres macrófitas evaluadas $(P>0.05)$, es decir, la riqueza de familias de macroinvertebrados, no está fuertemente influenciada por el tipo de macrófita (Figura 03).

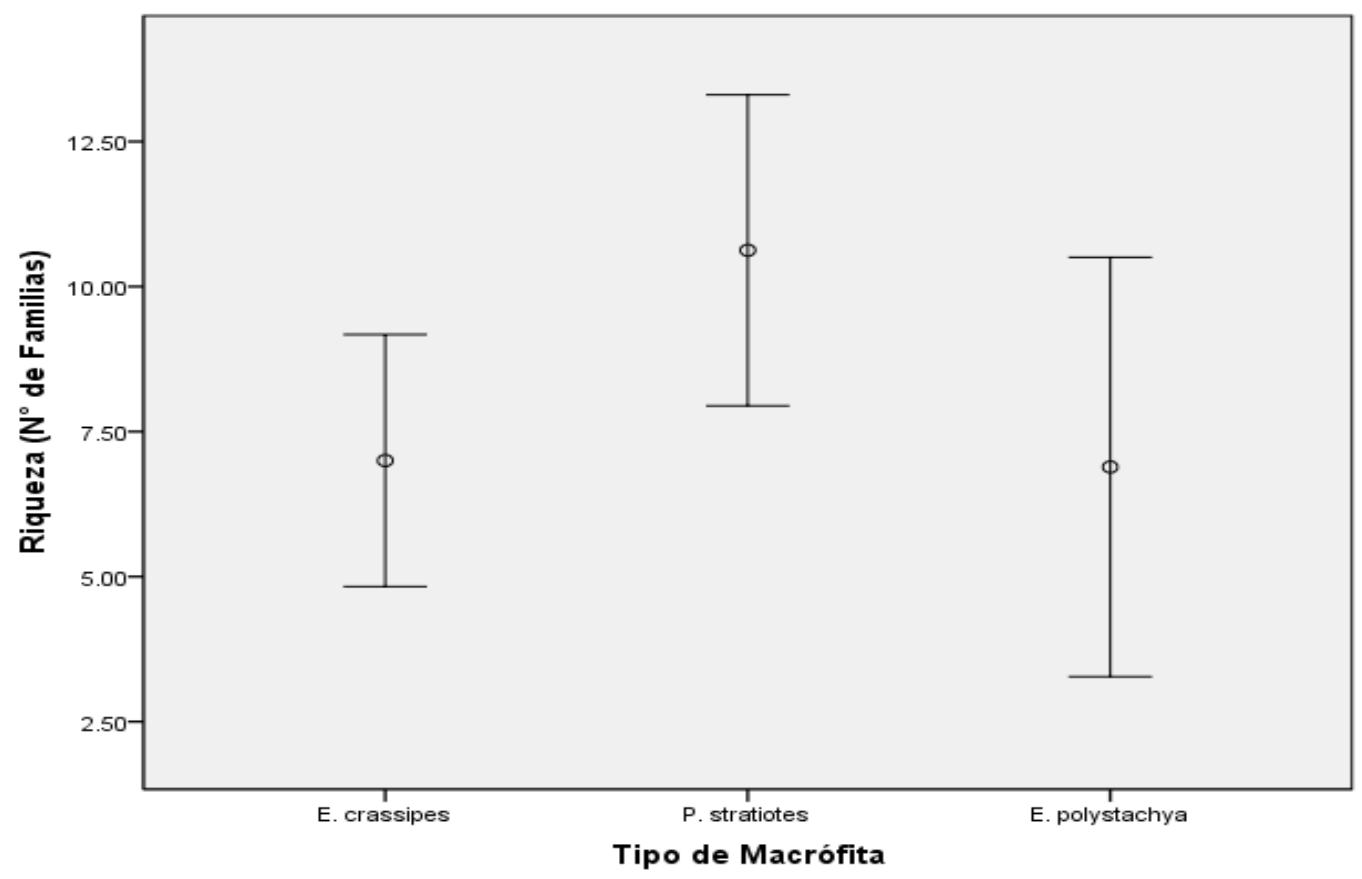

Figura 03. Riqueza de familias de macroinvertebrados acuáticos asociados a tres macrófitas en la laguna de Moronacocha (Iquitos- Perú). 


\section{Abundancia de macroinvertebrados acuáticos}

Se colectaron en total 1,539 individuos de macroinvertebrados acuáticos, destacándose la Clase Insecta por presentar la mayor abundancia de estos organismos en las tres macrófitas evaluadas ( $P$. stratiotes $=445, E$. crassipes $=368$ y $E$. polystachya $=298)$, seguida por la clase Oligochaeta $(P$. stratiotes $=62, E$. crassipes $=63$ y $E$. polystachya $=123)$, Gasterópoda ( $P$. stratiotes $=145)$, Branquiopoda $(P$. stratiotes $=16, E$. crassipes $=2$ y $E$. polystachya $=2)$ e Hirudinea $(P$. stratiotes $=5$, E. crassipes $=3$ y E. polystachya=6).

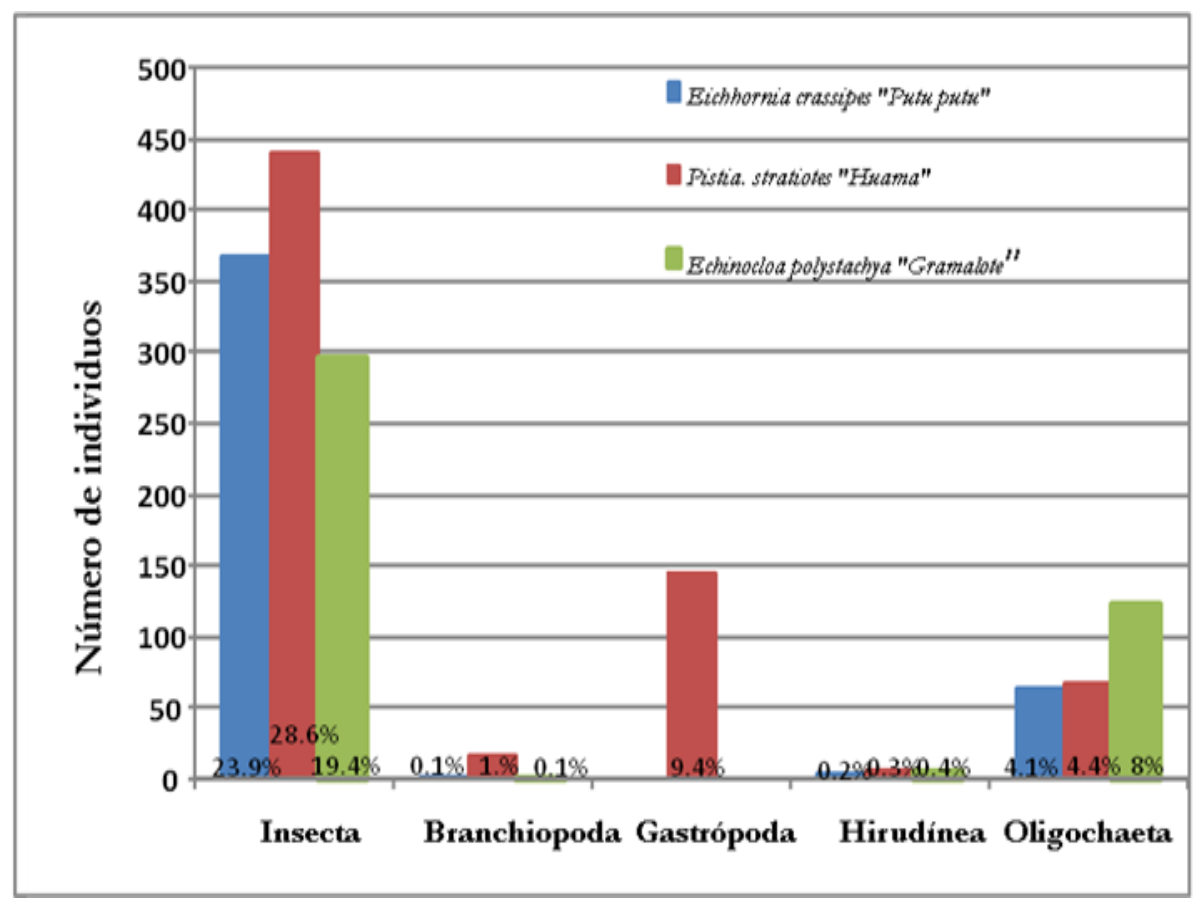

Figura 04. Abundancia total de macroinvertebrados acuáticos por clase taxonómica y tipo de macrófita en la laguna de Moronacocha (Iquitos- Perú).

\section{Abundancia de familias de macroinvertebrados acuáticos asociados a macrófitas}

De las 25 familias de macroinvertebrados reportadas para $P$. stratiotes solo seis: Planorbidae $\mathrm{N}=$ $145(21.8 \%)$, Hidrophilidae $\mathrm{N}=99$ (14.9\%), Chironomidae $\mathrm{N}=79(11.9 \%)$, Noteridae $\mathrm{N}=75$ (11.3\%), Haplotaxidae $\mathrm{N}=62(9.3 \%)$ y Elmidae $\mathrm{N}=51(7.7 \%)$ se presentaron abundantes en comparación con el resto de familias. En el caso de $E$. crassipes de un total de 21 familias reportadas, solo las familias Chironomidae con $\mathrm{N}=152(35 \%)$, seguido de la familia Noteridae con $\mathrm{N}=86(19.8 \%)$ y Haplotaxidae con $\mathrm{N}=63(14.5 \%)$, fueron las más abundantes. $\mathrm{Y}$ finalmente en el caso de $E$. polystachya, de un total de 25 familias de macroinvertebrados reportados, las familias Haplotaxidae con $\mathrm{N}=123(28.3 \%)$, Noteridae con $\mathrm{N}=108(24.8 \%)$ y Chironomidae con $\mathrm{N}=56$ (12.9\%) se destacaron por presentar la mayor abundancia.

\section{Ocurrencia de taxas de macroinvertebrados en las tres macrófitas acuáticas}

De 31 familias reportadas en este estudio, 17 estuvieron presentes en las tres macrófitas evaluadas (Dytiscidae, Elmidae, Noteridae, Scirtidae, Hydrophilidae, Chironomidae, Caenidae; Corixidae, Belostomatidae, Coenagrionidae, Libellulidae, Hidroptilidae, Polycentropodidae, Pyralidae, Limnadiidade, Glossiphonidae y Haplotaxidae) (Figura 05). 


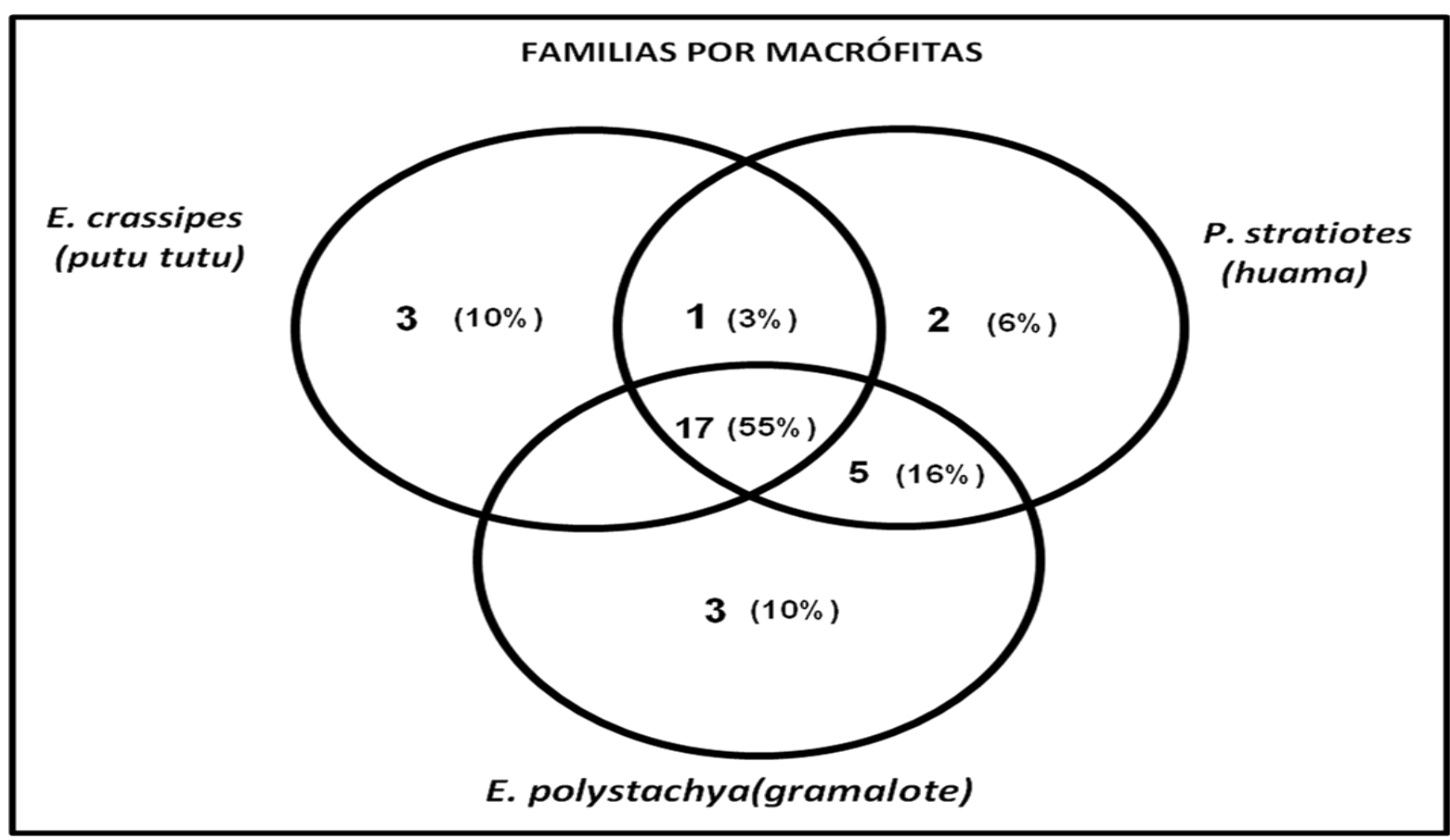

Figura 05. Número de familias de macroinvertebrados presentes en las tres macrofitas

La presencia de familias exclusivas en $E$. crassipes estuvo conformado solo por tres familias (Gyrinidae, Tricorythidae y Psychomyiidae), al igual que E. polystachya (Neoepheneridae, Polymitarcidae y Lestidae), mientras que $P$. stratiotes presentó solo dos familias exclusivas (Planorbidade y Ephydridae). El resto de familias ocurrieron en más de una macrófita.

\section{DISCUSIÓN}

En el presente estudio, las técnicas de ordenación y clasificación utilizadas, permitieron identificar las comunidades de macroinvertebrados en la laguna de Moronacocha, la riqueza de taxones observadas en este estudio son similares en cuanto a su composición taxonómica a las reportadas en otras cuencas hidrográficas de la Patagonia, Argentina (Miserendino, 2001) siendo los órdenes Ephemeroptera, Plecoptera, Trichoptera y Díptera, los más diversos y con mayor abundancia (Wais, 1987), la riqueza dominada por la phyla Artrópoda en la laguna de Moronacocha que presenta mayor número de larvas en la clase de los insectos, entre éstos, las órdenes que destacaron fueron: Coleóptera, Díptera, Ephemeróptera, Heteróptera y Odonata, similar al estudio realizado en las cinco localidades del Bajo Urubamba en donde también reporta que la mayor riqueza la presenta la phyla Arthropoda, dominada por larvas y adultos de insectos acuático y que las órdenes más representativas son Trichoptera, Coleóptera y Ephemeróptera, la presencia de mayor número de larvas en que adquieren los nutrientes y algunos micronutrientes de la columna del agua $y$, explica que la riqueza puede estar influenciada por las macrófitas que actúan como sustratos potencialmente estables para la colonización de larvas de macroinvertebrados, ya que dichas plantas propician una mayor disponibilidad de micro-hábitats y una diversidad de ítems alimenticios (Collier y Wilcock, 1998).

La macrofauna de la laguna de Moronacocha comprende un total de 1538 individuos, distribuidos en 31 familias, la mayor abundancia de familias se encontraron, en primer lugar Chironimidae (Díptera), segundo Noteridae (Coleóptera) y tercero haplotaxidae (Haplotaxida), de las 31 familias reportadas en el presente estudio, 21 están presentes en el putu putu y 25 familias en la huama y el gramalote, además se observa que 17 familias fueron ocurrentes en las tres especies de macrófitas, lo que demuestra que la mayor parte de las familias se encuentran compartidas y solo algunos macroinvertebrados son exclusivos en algunas macrófitas, debido a que los macroinvertebrados se pueden adaptar al sistema radicular de cualquier de las tres macrófitas, la 
presencia de los macroinvertebrados en las tres especies de macrófitas, muestran que la riqueza y abundancia son homogéneos, estadísticamente no presenta diferencias significativas, sin embargo si se estudian los macroinvertebrados en diferentes tipos de hábitat, se podrían encontrar divergencias en relación a la riqueza y abundancia, referente a la macrófita Huama, que presenta una ligera tendencia de albergar más organismos, posiblemente que estos han desarrollado especiales adaptaciones frente a los fuertes gradientes de humedad, temporalidad y oxígeno, la composición y la estructura de las comunidades varía temporal y espacialmente en forma muy significativa, incluso dentro de un mismo sistema y según el área cubierta por la vegetación (Paporello, 1987).

Referente a los parámetros físico-químico encontrados en la laguna de Moronacocha, la temperatura oscila entre $25.5^{\circ} \mathrm{C}-27.7^{\circ} \mathrm{C}$, con un promedio de $26.3^{\circ} \mathrm{C}$, no presentando mayores fluctuaciones y considerándose dentro de los parámetros normales para un cuerpo de agua léntico amazónico, el factor que contribuye para la riqueza y composición de especies de macroinvertebrados acuáticos es la temperatura, siendo influenciada éste por factores como la radiación solar y la precipitación fluvial, estas variaciones que se presentan corresponde a cambios normales, los cuales acompañan el ciclo hidrológico según Jacobsen (1997), el Oxigeno disuelto fluctuó entre $0,12 \mathrm{mg} / \mathrm{ml}-3.9 \mathrm{mg} / \mathrm{ml}$ con un promedio de $2.3 \mathrm{mg} / \mathrm{ml}$, uno de los factores determinantes en la presencia de bajas concentraciones de oxigeno se puede atribuir a las macrófitas, las que fijan en sus raíces numerosas colonias de microorganismos y el pH oscila entre 3.5 - 8.92 con un promedio de 6.7, debiéndose mantener el promedio arriba de 6 para que el nitrógeno durante el proceso de desnitrificación no sea liberado en forma de óxido nitroso y con ello disminuir el efecto invernadero.

En general las características físicos-químicos de la laguna, pueden estar influenciadas por los cambios climáticos que comprometen la sobrevivencia de la comunidad de macroinvertebrados.

\section{CONCLUSIONES}

- En las tres macrófitas evaluadas, la clase Insecta fue la más representativa en riqueza ( $P$. stratiotes $=21, E$. crassipes $=18$ y $E$. polystachya $=22$ ) y abundancia $(P$. stratiotes $=445, E$. crassipes $=368$ y $E$. polystachya $=298$ ) de familias de macroinvertebrados acuáticos en el Lago Moronacocha.

- Las familias más abundantes en las tres macrófitas evaluadas fueron Chironomidae, Noteridae y Haplotaxidae. Hydrophilidae, Elmidae y Planorbidae también fueron familias abundantes, pero solo para $P$. stratiotes.

- De 31 familias reportadas en este estudio, 17 estuvieron presentes en las tres macrófitas evaluadas, mientras que 3 fueron exclusivas para $E$. crassipes y $E$. polystachya y solo 2 exclusivas para $P$. stratiotes. El resto de familias ocurrieron en más de una macrófita.

- No se encontraron diferencias estadísticas significativas ( $P>0.05)$, en cuanto la riqueza y abundancia de macroinvertebrados acuáticos entre las tres macrófitas evaluadas.

- Los valores promedio de $\mathrm{T}^{\circ}\left(26.4^{\circ} \mathrm{C}\right)$ y $\mathrm{pH}(6.7)$ obtenidos de los puntos de colecta de cada tipo de macrófita se encontraron dentro de los límites adecuados, mientras que el nivel promedio de $\mathrm{O}_{2}(2.3 \mathrm{mg} / \mathrm{ml})$ se presentó muy bajo.

\section{AGRADECIMIENTOS}

Nuestro sincero agradecimiento a la Blga. Marianela Cobos Ruíz M.Sc. y a la Universidad Científica del Perú, por la oportunidad que nos dan para difundir nuestro trabajo. 


\section{REFERENCIAS BIBLIOGRÁFICAS}

ARAUJO.J. 2003. Evaluación de las comunidades fitoplanctónicas en la laguna de Moronacocha durante el periodo de creciente. Tesis para Optar el título de Biólogo. Universidad Nacional de la Amazonía Peruana - UNAP. Iquitos, Perú.

CAREY P. A., 1979, Desmidias del Lago Moronacocha - Estudio morfo-taxonómico del género Euastrum. Revista Conocimiento volumen 1: pp.16-26.

COLLIER KJ, RJ WILCOCK, 1998, As Meredith. Influence of substrate type and physicochemical conditions on Macroinvertebrate faunas and biotic indices of some lowland Waikato, New Zealand, streams. New Zealand Journal of Marine and Freshwater Research, Vol. 32: pp 1-19.

ENCARNACIÓN, F. 1985, Introducción a la Flora y a la Vegetación de la Amazonía Peruana: estado actual de los estudios, medio natural y ensayo de una clave de determinación de las formaciones vegetales en la llanura amazónica. Candollea 40: pp. 237- 252

JACOBSEN, L.; PERROW, M.; LANDKILDEHUS, F.; HJORNE, M.; LAURIDSEN, T. \& S. BERG. 1997. Las interacciones entre especies piscívoras, zooplanctívoros y zooplancton en submergedmacrophytes: observaciones preliminares de la caja y el estanque experiments. Hydrobiología 342/343: pp. 197-205.

McCAFFERTY, W. P., PROVONSHA, A. W. 1981. Aquatic Entomology. Jones and Bartlett Publishers, INC. Boston, $448 \mathrm{p}$.

MERRITT RW, CUMMINS KW. 1984, An introduction to the aquatic insects of North America. Second ed. Kendall/Hunt Publishing Company, Dubuque, Iowa, p. 722

MISERENDINO M (2001) Macroinvertebrate assemblages in Andean Patagonian rivers and streams: Environmental relationships. Hydrobiologia 444: 147-158.

PAPORELLO DE AMSLER G. 1987, Fauna asociada a las raíces de Eichhornia crassipes en una laguna del valle aluvial del Río Paraná ('Los Matadores', Santa Fé, Argentina). Revista de la Asociación de Ciencias Naturales del Litoral 1987b;18(1): 93-103.

ROLDÁN, G. P. 1996. Guía para el estudio de los macroinvertebrados acuáticos del departamento de Antioquia. Editorial Presencia Ltda. Bogotá, Colombia, 217 p.

MISERENDINO M \& L PIZZOLÓN 2004, Interactive effects of basin features and land-use change on macroinvertebrates communities of headwater streams in the Patagonian Andes. River Research and Applications 20: pp. 967-983.

TACHET, H RICHOUX, P., BOURNAUD, M Y USSEGLIO-POLATERA, P 2000. Invertebres d'eau douce, sistématique, bio-ecologie. CNRS. Editions. París. 588 pp

WAIS I. 1987, Macrozoobenthos of Negro River Basin, Argentine, Patagonia. Studies on Neotropical Fauna \& Environment 22: pp. 73-91

WAIS I \& A BONNETO 1988, Analysis of the allochthonous organic matter and associated macroinvertebrates in some streams of Patagonia (Argentina). Verhandlungen der Internationale Vereinigung fur Theoretische und Angewandte der Limnologie 23: pp. 14551459.

Recibido: 10 agosto 2011 / Aceptado: 20 octubre 2011 